\title{
CXCR3 alleviates renal ischemia-reperfusion injury via increase of Tregs
}

\author{
WENJING XIAN $^{1 *}$, JIALI WU ${ }^{2 *}$, QINGSHU LI $^{3}$, XUNSONG DU ${ }^{1}$, \\ NA WANG ${ }^{1}$, DAIYU CHEN ${ }^{1}$, WUXI GAO ${ }^{1}$ and JUN CAO $^{1}$ \\ ${ }^{1}$ Department of Anesthesiology, The First Affiliated Hospital of Chongqing Medical University, Chongqing 400016; \\ ${ }^{2}$ Department of Anesthesiology, The Affiliated Hospital of Southwest Medical University, Luzhou, Sichuan 646000; \\ ${ }^{3}$ Department of Pathology, Molecular and Cancer Research Center, Chongqing Medical University, \\ Chongqing 400016, P.R. China
}

Received September 28, 2020; Accepted March 19, 2021

DOI: $10.3892 / \mathrm{mmr} .2021 .12180$

\begin{abstract}
Increasing evidence has demonstrated that regulatory T cells (Tregs) suppress innate immunity, as well as protect the kidneys from ischemia-reperfusion injury (IRI) and offer a potentially effective strategy to prevent or alleviate renal IRI. The present study explored whether C-X-C motif chemokine receptor 3 (CXCR3) alleviated renal IRI by increasing Tregs. Male C57BL/6J mice were divided into sham-surgery, IRI, CXCR3 overexpression (OE-CXCR3)+IRI, PC61+IRI and OE-CXCR3+PC61+IRI groups. Histopathological examination of the kidney was carried out using hematoxylin-eosin and Masson staining. The levels of serum creatinine (Scr) and blood urea nitrogen (BUN) were measured. Blood and kidney levels of IL-6, TNF- $\alpha$, C-C motif chemokine ligand (CCL)-2 and IL-10 were detected by ELISA and western blotting. The levels of superoxide dismutase (SOD), glutathione peroxidase (GSH-Px) and malondialdehyde (MDA) in kidney tissues were also measured to assess oxidative stress. The population of Tregs in the kidney was assessed using flow cytometry. The results demonstrated that administration of OE-CXCR3 to IRI mice significantly decreased the levels of Scr, BUN, IL-6, TNF- $\alpha$, CCL-2 and MDA, increased the levels of IL-10, SOD and GSH-Px, and mitigated the morphologic injury and fibrosis induced by IR compared with the IRI group. In addition, administration of OE-CXCR3 induced significant reductions in the expression levels of fibrosis-related markers, including fibronectin and type IV collagen, and increased the number of Tregs. These roles of OE-CXCR3 were significantly
\end{abstract}

Correspondence to: Dr Jun Cao, Department of Anesthesiology, The First Affiliated Hospital of Chongqing Medical University, 1 Youyi Road, Yuzhong, Chongqing 400016, P.R. China

E-mail: caojun@hospital.cqmu.edu.cn

${ }^{*}$ Contributed equally

Key words: C-X-C motif chemokine receptor 3, regulatory $\mathrm{T}$ cells, inflammation, fibrosis, ischemia-reperfusion injury neutralized following deletion of Tregs with PC61 (anti-CD25 antibody). Together, the present study demonstrated that injection of OE-CXCR3 lentiviral vectors into animal models can alleviate renal IRI by increasing the number of Tregs. The results may be a promising approach for the treatment of renal IRI.

\section{Introduction}

Renal ischemia-reperfusion injury (IRI) is a common cause of acute kidney injury (AKI) and a crucial factor for the clinical outcome of kidney transplantation $(1,2)$. AKI progression frequently produces multiorgan failure and sepsis, resulting in increased morbidity and mortality in hospitalized patients (3). Therefore, it is necessary to develop effective methods to prevent or alleviate renal IRI.

Renal IRI is an acute inflammatory disease, in which immune cells serve crucial roles (4). Regulatory $\mathrm{T}$ cells (Tregs), a main $\mathrm{CD}^{+} \mathrm{T}$ lymphocyte subset, are characterized by the expression of cluster of differentiation (CD) 4 and CD25 on the cell surface, and upregulation of the expression level of forkhead box P3 (FoxP3) (5). Evidence has demonstrated that Tregs serve important roles in inflammation and maintaining immunological balance (6). The number of Tregs is dramatically decreased in a variety of autoimmune and inflammatory diseases, including multiple sclerosis, systemic lupus erythematosus, diabetes, rheumatoid arthritis, inflammatory bowel disease, psoriasis and atherosclerosis $(7,8)$. The removal of Tregs leads to the occurrence of immune dysregulation, enteropathy and X-linked syndrome $(9,10)$. In addition, Tregs are closely implicated in the pathogenesis of ischemic AKI, in which Tregs suppress innate immunity and protect against renal IRI (11-13). Increasing the number of Tregs is a potential effective strategy to prevent or alleviate renal IRI (11-13).

CXC chemokine receptor 3 (CXCR3), also called G-protein-coupled receptor 9 or CD183, is mainly expressed in T cell natural killer cells (14). Hasegawa et al (15) transferred CXCR3-overexpressed Tregs into recipient mice and found the recipient mice demonstrated significant amelioration of graft-vs-host disease (GVHD) changes in the liver, lung and intestine. Oo et al (16) isolated $\mathrm{CD}^{+} \mathrm{CD} 25^{+} \mathrm{CD} 127^{\text {low }} \mathrm{FoxP} 3^{+}$ 
Tregs from chronically inflamed human liver and found that these Tregs expressed higher levels of the chemokine receptors CXCR3 and CCR4 compared with blood-derived Tregs and liver-derived Tregs. Their study proposed that CXCR3 and CCR4 recruited Tregs to sites of inflammation in patients with chronic hepatitis. Similarly, our previous study reported that CXCR3 expression on Tregs was elevated $72 \mathrm{~h}$ post-reperfusion and was inversely correlated with blood urea nitrogen (BUN), serum creatinine (Scr) and kidney injury, suggesting a protective role of CXCR3 in renal IR (17). However, the roles of CXCR3 in regulation of Tregs number and alleviation of renal IRI remain to be elucidated.

The present study aimed to discover whether CXCR3 serves a protective role in renal IRI via increasing the number of Tregs.

\section{Materials and methods}

Animals and grouping. A total of 45 male C57BL/6J mice (age, 8-12 weeks; weight, 20-25 g) were purchased from Vital River Laboratories. The animals were fed with common feed and sterile water ad libitum in a specific pathogen-free laboratory, and housed at $22 \pm 1^{\circ} \mathrm{C}$ with $55 \pm 1 \%$ humidity under a $12-\mathrm{h}$ light/dark cycle. Animals were accommodated for 1 week before experiments.

The mice were randomly divided into the sham-surgery, IRI, CXCR3 overexpression (OE-CXCR3)+IRI, PC61+IRI and OE-CXCR3+PC61+IRI groups (n=15 mice/group).

Animal experiments were carried out at The First Affiliated Hospital of Chongqing Medical University (Chongqing, China) and were approved by the Animal Ethics and Use Committee of the First Affiliated Hospital of Chongqing Medical University (approval no. 2017-028).

Renal IRI model establishment and treatments. Renal IRI models were established as previously described (17). In brief, mice were anaesthetized via intraperitoneal administration of $80 \mathrm{mg} / \mathrm{kg} 3 \%$ sodium pentobarbital. Ischemia of both kidneys was induced by clamping renal pedicles for 45 min with microvascular clamps followed by reperfusion. Animals in the sham-surgery group underwent the same surgical procedure without clamping of the renal pedicles. Animals in OE-CXCR3+IRI group were administered $100 \mu \mathrm{l}$ filter-purified OE-CXCR3 lentivirus vector $\left(\sim 5 \times 10^{5} \mathrm{IU} / \mu \mathrm{l}\right)$ via tail vein injection before reperfusion. Mice in the IRI group were given an equal volume of a filter-purified non-targeting lentivirus control (OE-NC), which served as a negative control. OE-CXCR3 and OE-NC were purchased from Shanghai GenePharma Co., Ltd. To deplete Tregs in vivo, $100 \mu \mathrm{g}$ PC61 (BioLegend) was given to mice via tail vein injection following reperfusion. Following anesthetization with $3 \%$ sodium pentobarbital $(80 \mathrm{mg} / \mathrm{kg})$, five mice from each group were decapitated to collect the blood samples after 24, 48 and $72 \mathrm{~h}$ of reperfusion. The kidneys were collected for further analysis, including reverse transcription-quantitative (RT-q) PCR, western blotting and histopathological examination.

Biochemical detection. The blood samples (1 ml) were centrifuged for $5 \mathrm{~min}$ at a speed of $978 \mathrm{x} \mathrm{g} / \mathrm{min}$ at $4^{\circ} \mathrm{C}$ to obtain the serum samples. Then, an automatic biochemistry analyzer
(Hitachi 7060; Hitachi) was used to detect the serum levels of BUN and Scr.

Histopathological examination. The right kidney was fixed in $4 \%$ formaldehyde for $24 \mathrm{~h}$ at room temperature, dehydrated with $95 \%$ ethanol and embedded in $55^{\circ} \mathrm{C}$ paraffin, and then cut into $4-\mu \mathrm{m}$ sections. The sections were stained with hematoxylin-eosin (HE) to assess the histopathological changes at $72 \mathrm{~h}$ post-reperfusion. Kidney injury was scored according to a previous study (18): 0, no damage; 1, mild damage, including rounded epithelial cells and dilated tubular lumen; 2, moderate damage, including substantially dilated lumen, flattened epithelial cells and nuclear staining loss; and 3 , severe damage, including destroyed tubules with no nuclear staining of epithelial cells.

To determine the areas of fibrotic kidney lesions, kidney tissue sections were stained with Masson trichrome staining (Beijing Solarbio Science \& Technology Co., Ltd.) based on the instructions provided by the manufacturer. Red-stained tissue indicated normal and blue-stained tissue indicated renal fibrotic tissue. The staining was captured using a light microscope (magnification, $\mathrm{x} 100$ ).

ELISA. The concentrations of IL-10 (cat. no. BMS614INST), IL-6 (cat. no. BMS603-2), TNF- $\alpha$ (cat. no. BMS607-3) and C-C motif chemokine ligand 2 (CCL-2; cat. no. BMS6005) in mouse blood samples after $72 \mathrm{~h}$ of reperfusion were measured using ELISA kits (Thermo Fisher Scientific, Inc.) based on the manufacturer's protocols. The absorbance at $450 \mathrm{~nm}$ for each sample was determined using an ELISA plate reader (BioTek China).

Measurement of superoxide dismutase (SOD), glutathione peroxidase (GSH-Px) and malondialdehyde (MDA). The kidney tissues were homogenized and centrifuged at $4{ }^{\circ} \mathrm{C}$ for $10 \mathrm{~min}$ at $10,000 \mathrm{x}$. Then, the activities of SOD, GSH-Px and MDA were determined using commercial kits (Beijing Solarbio Science \& Technology Co., Ltd.) based on the manufacturer's protocols and the OD values were determined at 560 (SOD), 412 (GSH-Px), 532 and 600 (MDA) nm, respectively, using a microplate reader.

Preparation of the single-cell suspensions from the kidneys. Single-cell suspensions of kidney cells were isolated from C57BL/6 mice based on a previous study (19). In brief, the kidney was triturated, sequentially passed through a 200-mesh sieve and lysed with a red blood cell lysis buffer (BioLegend). The cells were then incubated with $1.6 \mathrm{mg} / \mathrm{ml}$ collagenase I (Sigma-Aldrich; Merck KGaA) and $200 \mathrm{mg} / \mathrm{ml}$ DNase I (Sigma-Aldrich; Merck KGaA) in RPMI-1640 medium (Thermo Fisher Scientific, Inc.) for $30 \mathrm{~min}$ at $37^{\circ} \mathrm{C}$. Then, the cells were filtered by 70- and 40-mm mesh in turn and lysed with the red blood cell lysis buffers.

Flow cytometry. The number of Tregs was evaluated using flow cytometry. In brief, the aforementioned cells isolated from the kidneys were incubated with the anti-CD4 antibody (cat. no. 11-0041-82; FITC; eBioscience; Thermo Fisher Scientific, Inc.) at a dilution of $1: 10$ for $1 \mathrm{~h}$ at $4^{\circ} \mathrm{C}$, followed by incubation with Cytofix/Cytoperm (BD Biosciences) 
Table I. Primer sequences.

\begin{tabular}{lcc}
\hline Gene & \multicolumn{1}{c}{ Forward (5'-3') } & Reverse $\left(5^{\prime}-3^{\prime}\right)$ \\
\hline CXCR3 & AATGCCACCCATTGCCAGTA & TAGCTCGAAAACGCCTCTGG \\
KIM-1 & CAGGGTCTCCTTCACAGCAG & CCACCACCCCCTTTACTTCC \\
Nephrin & ATGGGAGCTAAGGAAGCCAC & ACTCGTACTCCGCATCATCG \\
GAPDH & AAGAGGGATGCTGCCCTTAC & TACGGCCAAATCCGTTCACA
\end{tabular}

CXCR3, C-X-C motif chemokine receptor 3; KIM-1, kidney injury molecule-1.

at $4^{\circ} \mathrm{C}$ for $15 \mathrm{~min}$ to permeabilize the cell membranes. The cells were then stained with PE-conjugated anti-Foxp3 antibodies (cat. no. 12-5773-82; eBioscience; Thermo Fisher Scientific, Inc.) at 1:20 dilution for $1 \mathrm{~h}$ at $4^{\circ} \mathrm{C}$ and analyzed on a BD FACSCalibur System (BD Bioscience). Tregs were considered as $\mathrm{CD}^{+}$and Foxp3 ${ }^{+}$and was analyzed using the FlowJo 7.6 software (FlowJo LLC).

$R T-q P C R$. Total RNA was extracted using TRIzol ${ }^{\circledR}$ reagent (Invitrogen; Thermo Fisher Scientific, Inc.) in accordance with the manufacturer's instructions. After quantification with a Nanodrop spectrophotometer (Thermo Fisher Scientific, Inc.), $1 \mu \mathrm{g}$ RNA sample was reverse transcribed into cDNA $\left(42^{\circ} \mathrm{C}\right.$, $\left.2 \mathrm{~min} ; 37^{\circ} \mathrm{C}, 15 \mathrm{~min} ; 85^{\circ} \mathrm{C}, 5 \mathrm{sec}\right)$ using a First Strand cDNA Synthesis kit purchased from CWBio. Subsequently, the RT-qPCR assay was performed based on the descriptions of a SYBR Premix Ex Taq II kit (Takara Biotechnology Co., Ltd.) in an ABI PRISM 7700 Sequence Detection System (Applied Biosystems; Thermo Fisher Scientific, Inc.). GAPDH was used as the internal reference to normalize mRNA expression levels. The calculation of mRNA levels was executed using the $2^{-\Delta \Delta C q}$ method (20). The qPCR thermocycling conditions were as follows: Initial denaturation at $95^{\circ} \mathrm{C}$ for $30 \mathrm{sec}$, followed by annealing and elongation for 39 cycles of $95^{\circ} \mathrm{C}$ for $15 \mathrm{sec}, 60^{\circ} \mathrm{C}$ for $30 \mathrm{sec}$ and $72^{\circ} \mathrm{C}$ for $30 \mathrm{sec}$, and a final extension at $72^{\circ} \mathrm{C}$ for $2 \mathrm{~min}$. The primer sequences used in the present study are shown in Table I.

Western blotting. Total protein was collected using radioimmunoprecipitation assay lysis buffer (Beyotime Institute of Biotechnology), supplemented with $1 \%(\mathrm{v} / \mathrm{v})$ protease inhibitors (Beyotime Institute of Biotechnology). Then, the protein concentrations were measured using BCA method (Thermo Fisher Scientific, Inc.). Subsequently, $30 \mu \mathrm{g}$ protein from each sample was separated by sodium dodecyl sulfate-polyacrylamide gel electrophoresis on $8 \%$ gels and then transferred onto polyvinylidene difluoride (PVDF) membranes (EMD Millipore). The PVDF membranes were incubated with $5 \%$ fat-free milk for $1 \mathrm{~h}$ at room temperature and then incubated with the primary antibodies overnight at $4^{\circ} \mathrm{C}$, including anti-fibronectin antibody (1:2,000 dilution; cat. no. ab2413; Abcam), anti-type IV collagen antibody (1:2,000 dilution; cat. no. ab19808; Abcam), anti-IL-6 antibody (1:2,000 dilution; cat. no. sc-32296; Santa Cruz Biotechnology, Inc.), anti-TNF- $\alpha$ antibody (1:2,000 dilution; ab183218; Abcam), anti-CCL-2 antibody (1:2,000 dilution; cat. no. 2029; Cell Signaling Technology, Inc.), anti-IL-10 antibody (1:4,000 dilution; ab189392; Abcam), anti-Bax antibody (1:3,000 dilution; cat.no. ab32503; Abcam), anti-Bcl-2 antibody (1:3,000 dilution; cat. no. ab182858; Abcam), anti-cleaved caspase-3 antibody (1:3,000 dilution; cat. no. ab49822; Abcam) and anti- $\beta$-actin antibody (1:5,000 dilution; cat. no. AM4302; Thermo Fisher Scientific, Inc.). Next, the membranes were probed with horseradish peroxidase-conjugated secondary antibodies (1:5,000 dilution; cat. nos. ab6728 and ab ab6721; Abcam) for $1 \mathrm{~h}$ at room temperature. Protein signals were detected using iBright CL750 (Thermo Fisher Scientific, Inc.) after being visualized with ECL visualization reagent (Thermo Fisher Scientific, Inc.) at room temperature. The gray-scale value analysis was performed using ImageJ software (version 1.48; National Institutes of Health).

Statistical analysis. Data are expressed as the mean \pm standard deviation from $\geq 3$ experiments. Statistical analyses were performed using SPSS software (version 21.0; IBM Corp.). Two-sided unpaired t-tests and one-way ANOVA followed by Bonferroni post-hoc tests were used to compare differences between 2 and $\geq 3$ groups, respectively. Kidney injury score was expressed as median and range, and was analyzed using the Kruskal-Wallis test followed by the Steel-Dwass test. $\mathrm{P}<0.05$ was considered to indicate a statistically significant difference.

\section{Results}

Upregulation of CXCR3 alleviates kidney IRI. First, the effect of CXCR3 was assessed on the progression of kidney IRI. Compared with the IRI group, CXCR3 expression was significantly elevated in mouse kidney following OE-CXCR3 treatment (Fig. 1A). Upregulation of CXCR3 significantly decreased the blood levels of BUN and Scr induced by IR (Fig. 1B and C), decreased the expression levels of kidney injury molecule-1 (KIM-1) and increased Nephrin expression (Fig. 1D). HE staining demonstrated that no morphological change was apparent in the sham group. However, severe structural disruptions, such as tubular dilatation, loss of nuclear staining and brush border, and flattened renal epithelial cells were observed in the IRI group, whereas CXCR3 overexpression clearly mitigated the morphological injury induced by IR (Fig. 1E). In addition, the expression levels of Bax and cleaved caspase- 3 were elevated, and Bcl-2 expression was reduced in the IRI group compared with the control group, whereas these tendencies were mitigated by CXCR3 overexpression (Fig. 1F). These findings demonstrated that upregulation of CXCR3 alleviated kidney injury induced by IR. 
A

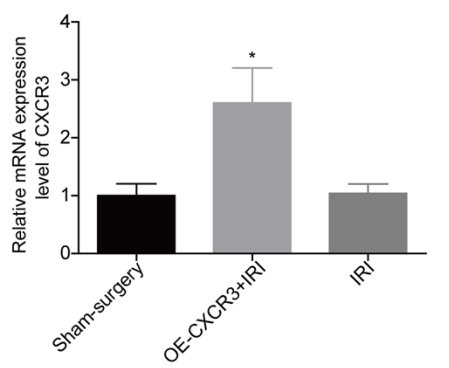

E

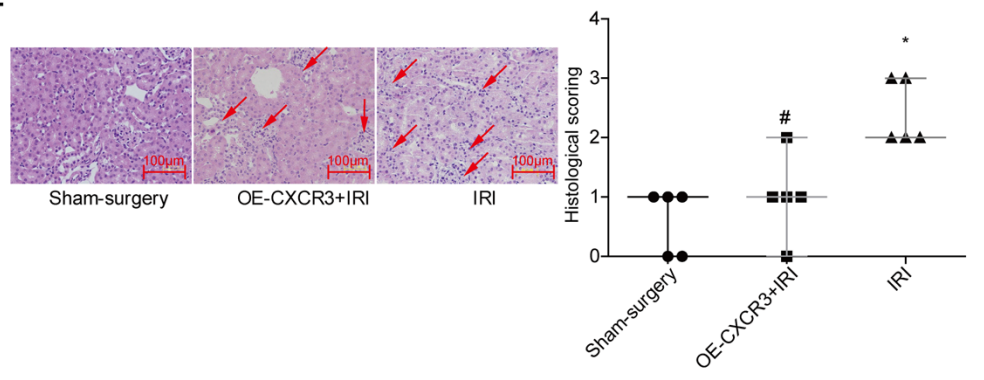

C

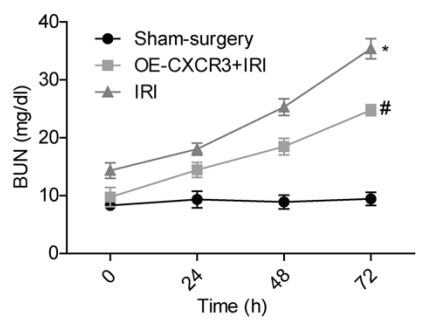

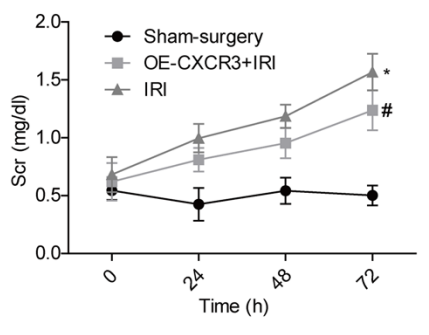

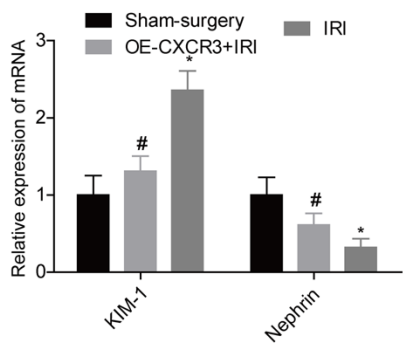

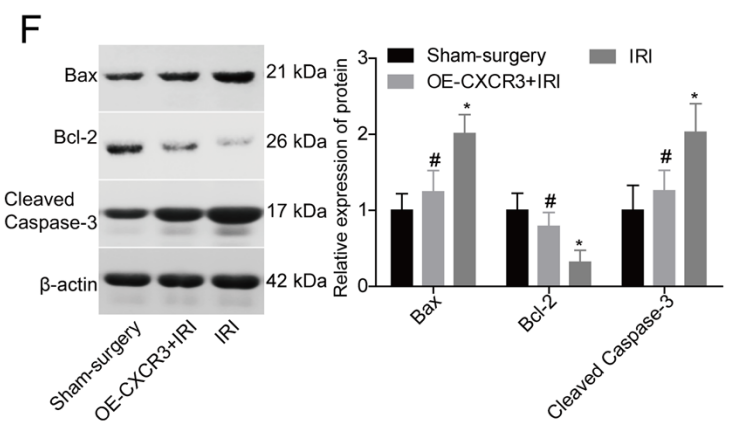

Figure 1.CXCR3 assuages kidney IRI. (A) RT-qPCR was used to analyze the mRNA expression levels of CXCR3 in mice kidney tissues from the sham-surgery, IRI and OE-CXCR3+IRI groups. Blood levels of (B) BUN and (C) Scr in mice of the sham-surgery, IRI and OE-CXCR3+IRI groups. (D) RT-qPCR was used to analyze the mRNA expression levels of KIM-1 and Nephrin in mice kidney tissues from the sham-surgery, IRI and OE-CXCR3+IRI groups. (E) Hematoxylin-eosin staining was used to assess the histopathological changes to the kidney, and the arrows indicated lesion sites (scale bar, $100 \mu \mathrm{m}$ ). Kidney injury score was expressed as median and range, with the longest line indicating the median. (F) Protein expression levels of Bax, Bcl-2 and cleaved caspase-3 in mice kidney were determined using western blotting. ${ }^{*} \mathrm{P}<0.05$ vs. sham-surgery group; ${ }^{\prime} \mathrm{P}<0.05$ vs. IRI group. CXCR3, C-X-C motif chemokine receptor 3; IRI, ischemia-reperfusion injury; RT-qPCR, reverse transcription-quantitative PCR; OE, overexpression; BUN, blood urea nitrogen; Scr, serum creatinine; KIM-1, kidney injury molecule-1.
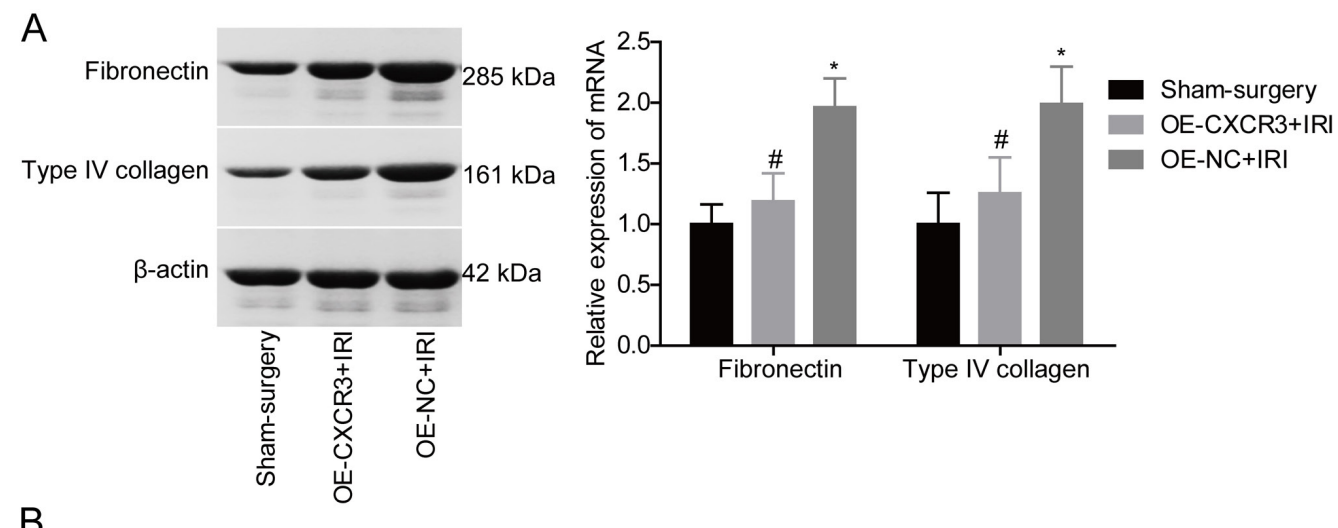

B

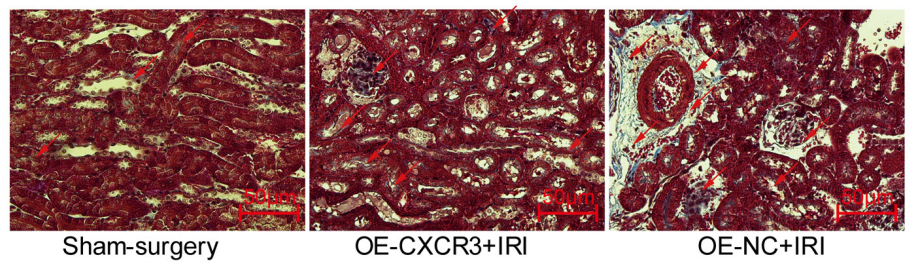

Figure 2. CXCR3 mitigates kidney fibrosis induced by IR. (A) Western blotting was performed to detect the protein expression levels of fibronectin and type IV collagen in mice kidney from the sham-surgery, IRI and OE-CXCR3+IRI groups. (B) Masson trichrome staining was used to assess renal fibrosis and the arrows indicated fibrosis sites (scale bar, $50 \mu \mathrm{m}$ ). ${ }^{*} \mathrm{P}<0.05$ vs. sham-surgery group; ${ }^{\sharp} \mathrm{P}<0.05$ vs. IRI group. CXCR3, C-X-C motif chemokine receptor 3 ; IR, ischemia-reperfusion; IRI, ischemia-reperfusion injury; OE, overexpression.

CXCR3 alleviates kidney fibrosis induced by IR. The effects of CXCR3 on kidney fibrosis induced by IR were also assessed. Compared with the sham group, the protein expression levels of fibronectin and type IV collagen were significantly increased, whereas CXCR3 overexpression significantly reduced their levels in kidney tissues (Fig. 2A). Masson trichrome staining demonstrated that CXCR3 overexpression markedly mitigated the kidney fibrosis induced by IR (Fig. 2B). These results 
A

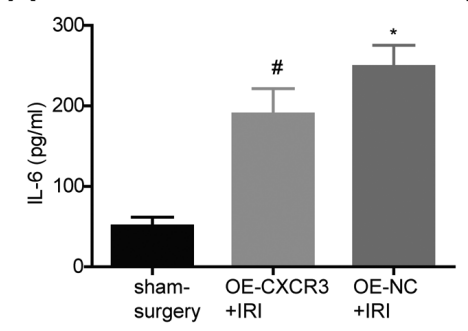

$\mathrm{D}$

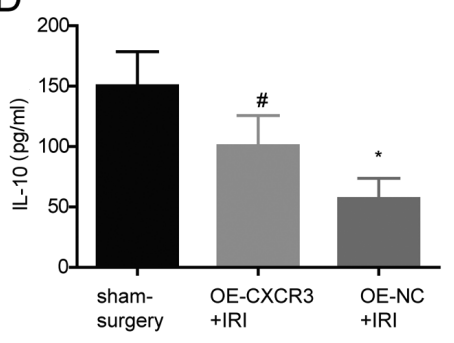

B

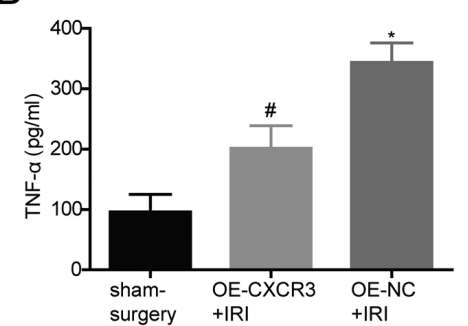

E

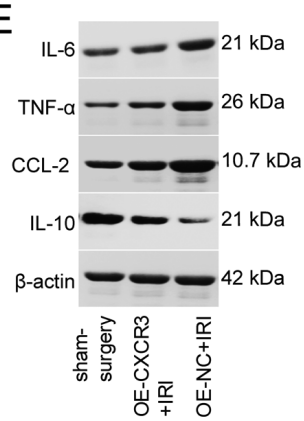

C
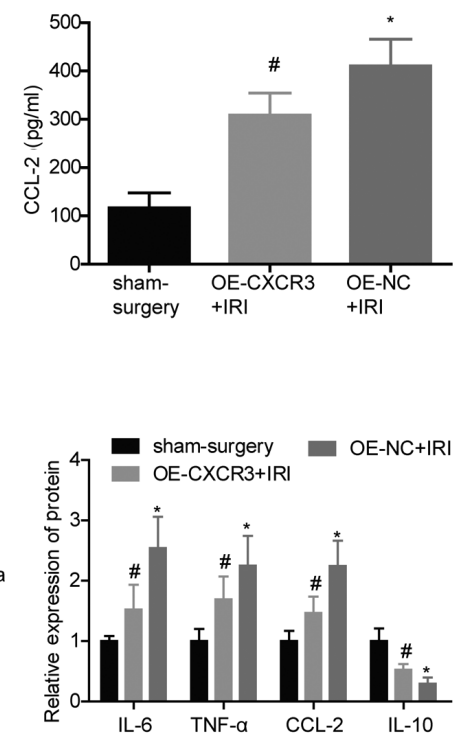

Figure 3. CXCR3 suppresses the enhanced inflammatory responses induced by IR. ELISA was used to detect the blood levels of (A) IL-6, (B) TNF- $\alpha$, (C) CCL-2 and (D) IL-10 in mice from the sham-surgery, IRI and OE-CXCR3+IRI groups. (E) Protein expression levels of IL-6, TNF- $\alpha$, CCL-2 and IL-10 in mice kidney were determined by western blotting. "P<0.05 vs. sham-surgery group; ${ }^{\#} \mathrm{P}<0.05$ vs. IRI group. CXCR3, C-X-C motif chemokine receptor 3 ; IR, ischemia-reperfusion; CCL-2, C-C motif chemokine ligand 2; IRI, ischemia-reperfusion injury; OE, overexpression.

A

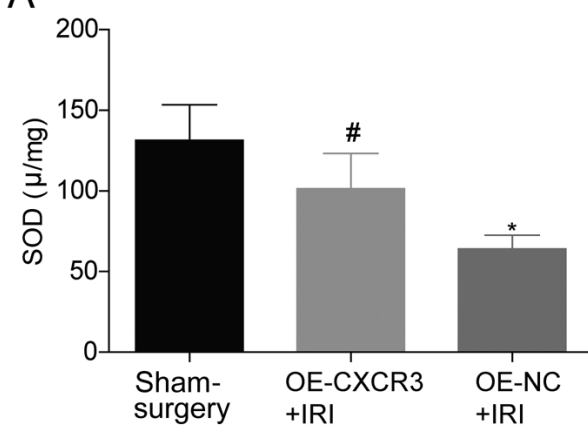

B

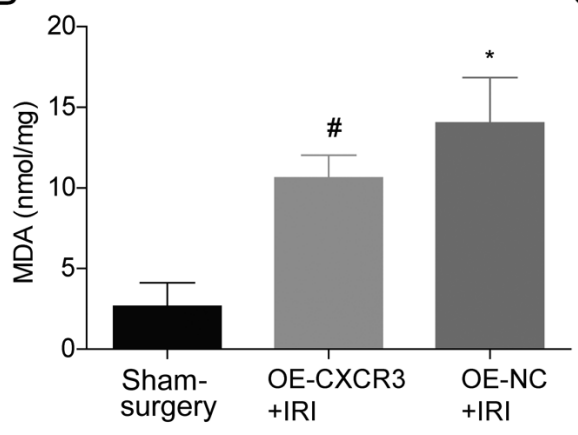

C

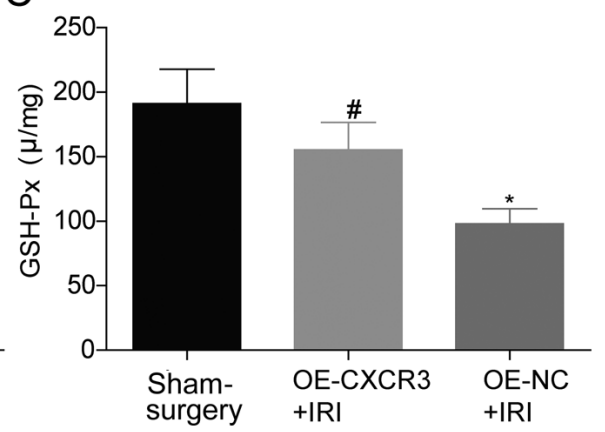

Figure 4. CXCR3 represses oxidative stress induced by IR. The kidney levels of (A) SOD, (B) MDA and (C) GSH-Px were measured using commercial kits. ${ }^{*} \mathrm{P}<0.05$ vs. sham-surgery group; ${ }^{\#} \mathrm{P}<0.05$ vs. IRI group. CXCR3, C-X-C motif chemokine receptor 3; IR, ischemia-reperfusion; SOD, superoxide dismutase; MDA, malondialdehyde; GSH-Px glutathione peroxidase.

demonstrated that upregulation of CXCR3 significantly lessened kidney fibrosis induced by IR.

CXCR3 suppresses the enhanced inflammatory responses and oxidative stress induced by IR. Compared with the sham-surgery group, the blood levels of IL-6, TNF- $\alpha$ and CCL-2 in the IRI group were significantly elevated, whereas IL-10 levels were decreased (Fig. 3A-D). However, overexpression of CXCR3 apparently decreased IL-6, TNF- $\alpha$ and CCL-2 levels, and increased IL-10 levels in the blood samples of mice induced by IR as compared with the IRI group (Fig. 3A-E).

The effect of CXCR3 on the oxidative stress induced by IR was also assessed. Compared with the sham-surgery group, SOD and GSH-Px levels in mice kidney tissues were significantly decreased, and MDA levels were increased in the IRI group, which were rescued by CXCR3 overexpression
(Fig. 4A-C). Taken together, these results illustrated that CXCR3 alleviated the inflammatory response and oxidative stress induced by IR in vivo.

CXCR3 increases the population of Tregs in mice kidney following IR. The single-cell suspensions from the kidneys were collected to analyze the effect of CXCR3 on the population of Tregs. Compared with the sham-surgery group, the population of Tregs was significantly increased, which was further increased following CXCR3 overexpression (Fig. 5). This result indicated that CXCR3 increased the population of Tregs in mice kidney following IR.

Deletion of Tregs abrogates the role of CXCR3 in improving kidney IRI. Next, it was explored whether Tregs were involved in CXCR3-mediated alleviation of kidney injury induced by 


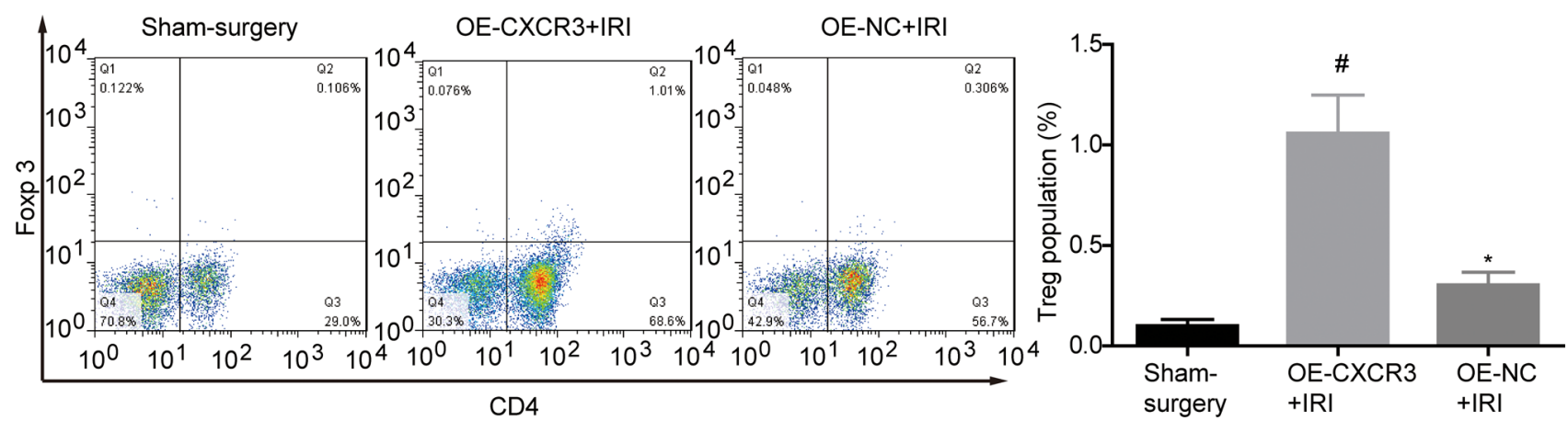

Figure 5. CXCR3 increases the population of Tregs in mice kidneys following IR. Flow cytometry was used to determine the population of Tregs in kidney from sham-surgery, IRI and OE-CXCR3+IRI groups. " $\mathrm{P}<0.05$ vs. sham-surgery group; ${ }^{*} \mathrm{P}<0.05$ vs. IRI group. CXCR3, C-X-C motif chemokine receptor 3 ; Tregs, regulatory T cells; IR, ischemia-reperfusion; IRI, ischemia-reperfusion injury; OE, overexpression.

A
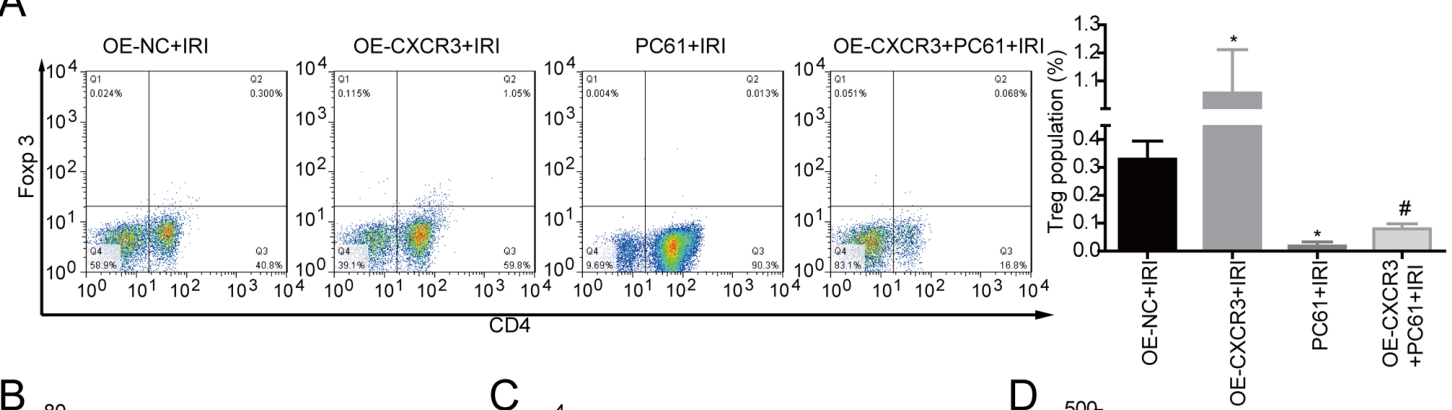

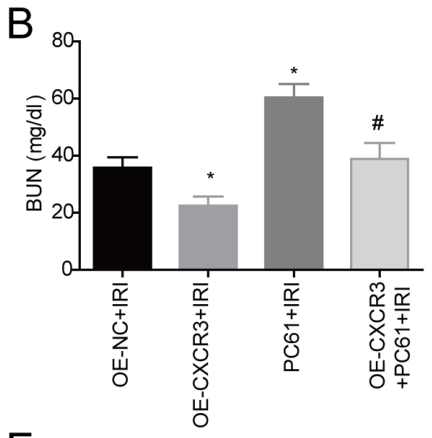

$\mathrm{E}$

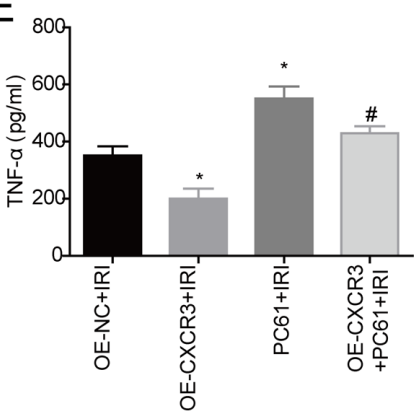

C

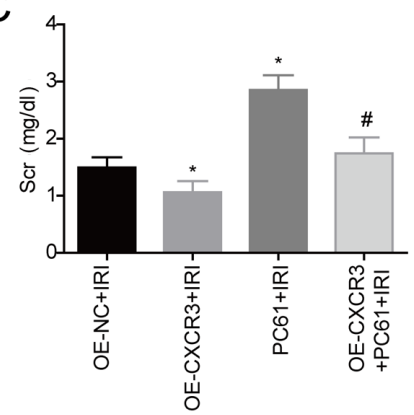

$\mathrm{F}$

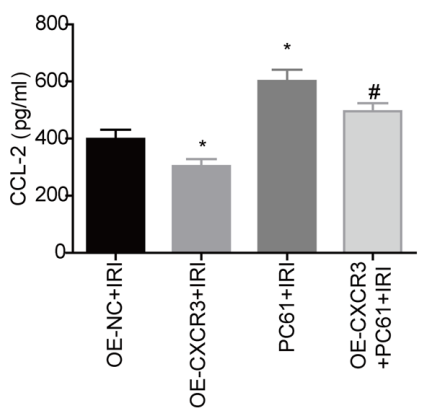

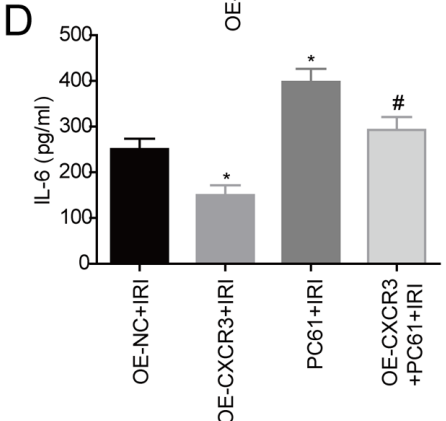

G

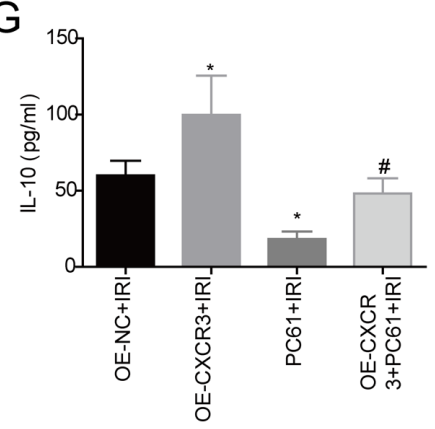

Figure 6. Deletion of Tregs abrogates the role of CXCR3 in improving kidney inflammation. Samples from mice in the IRI, OE-CXCR3+IRI, PC61+IRI and OE-CXCR3+PC61+IRI groups were assessed. (A) Flow cytometry was used to determine the population of Tregs in kidney cells. The blood levels of (B) BUN and (C) Scr were tested. ELISA was used to detect the blood levels of (D) IL-6, (E) TNF- $\alpha$, (F) CCL-2 and (G) IL-10. "P<0.05 vs. IRI group; ${ }^{*} \mathrm{P}<0.05$ vs. OE-CXCR3+IRI group. Tregs, regulatory T cells; CXCR3, C-X-C motif chemokine receptor 3; IRI, ischemia-reperfusion injury; OE, overexpression; BUN, blood urea nitrogen; Scr, serum creatinine; CCL-2, C-C motif chemokine ligand 2.

IR. The population of Tregs in kidney tissues was significantly decreased when the mice were treated with PC61 (Fig. 6A). The blood levels of BUN, Scr, IL-6, TNF- $\alpha$ and CCL-2 were significantly increased, and IL-10 levels were decreased in the OE-CXCR3+PC61+IRI group as compared with the OE-CXCR3+IRI group (Figs. 6B-G and 7D). In addition, deletion of Tregs worsened the kidney fibrosis and morphological injury induced by IR in PC61+IRI group compared with the IRI group, or in OE-CXCR3+PC61+IRI group compared with the OE-CXCR3+IRI group (Fig. 7A-C). These findings demonstrated that CXCR3 mitigated kidney IRI by increasing the population of Tregs. 
A
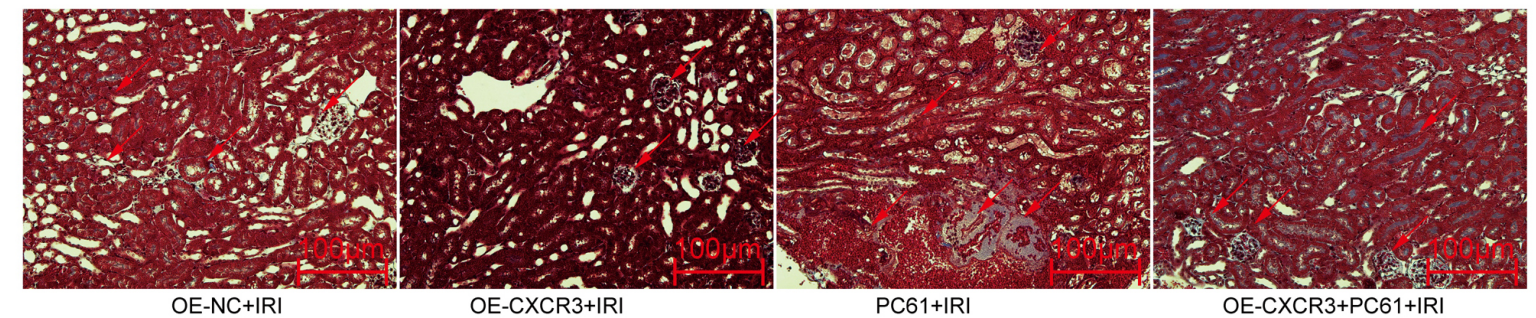

B
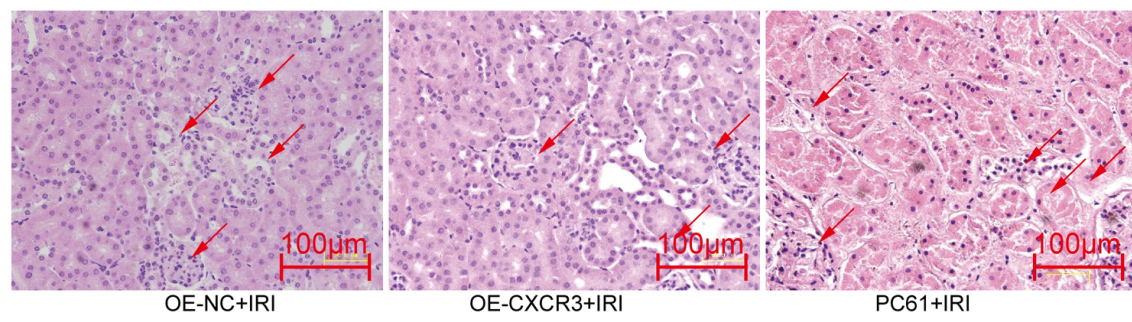

OE-CXCR3+PC61+|RI

C

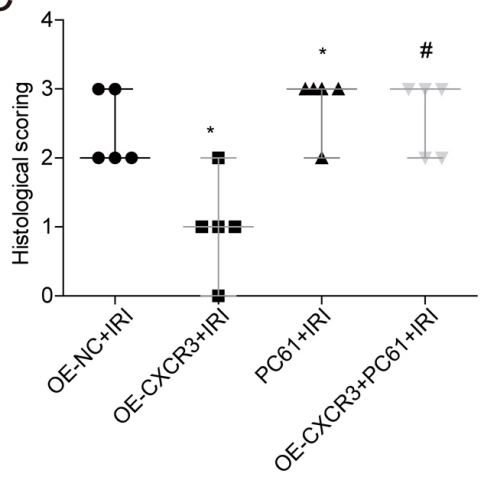

D

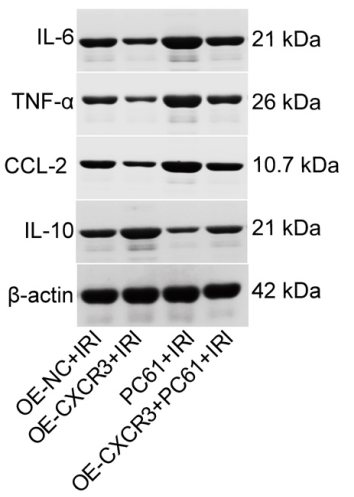

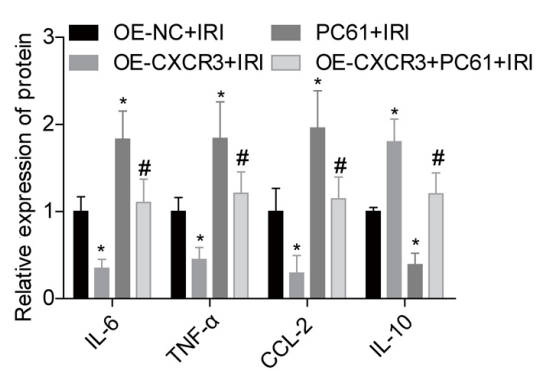

Figure 7. Deletion of Tregs abrogates the role of CXCR3 in improving kidney IRI. Samples from mice in the IRI, OE-CXCR3+IRI, PC61+IRI and OE-CXCR3+PC61+IRI groups were assessed. (A) Masson trichrome staining was used to assess renal fibrosis, and the arrows indicated fibrosis sites (scale bar, $100 \mu \mathrm{m}$ ). (B and C) Hematoxylin-eosin staining was used to assess the histopathological changes in kidneys, and the arrows indicated lesion sites (scale bar, $100 \mu \mathrm{m}$ ). Kidney injury score was expressed as median and range, with the longest line indicating the median. (D) Protein expression levels of IL-6, TNF- $\alpha$, CCL-2 and IL-10 in mice kidneys were determined by western blotting. ${ }^{*} \mathrm{P}<0.05$ vs. IRI group; ${ }^{\text {"P }}<0.05$ vs. OE-CXCR3+IRI group. CXCR3, C-X-C motif chemokine receptor 3; IRI, ischemia-reperfusion injury; OE, overexpression; CCL-2, C-C motif chemokine ligand 2.

\section{Discussion}

The present study explored whether CXCR3 was involved in kidney IRI via modulating the number of Tregs. The results illustrated that overexpression of CXCR3 in IR mice significantly mitigated IR-mediated kidney injury, inflammatory reactions, fibrosis, oxidative stress and increased the number of Tregs. However, deletion of Tregs with PC61 apparently impaired the beneficial effects of CXCR3, suggesting that CXCR 3 alleviated renal IRI via expansion of Tregs.

The role of CXCR3 in kidney IRI is controversial. Fiorina et al (21) reported that CXCR3-deficient mice $\left(\mathrm{CXCR} 3^{-/}\right)$presented with lower serum creatinine levels, improved survival rate, and less acute tubular necrosis and cellular infiltrates in renal IR models, accompanied with decreased Th1 population. Zhang et al (22) found that rapamycin significantly improved renal function and ameliorated histological injury by increasing the $\mathrm{CXCR}^{+}$natural killer $\mathrm{T}$ cell population in the peripheral blood and kidneys. We previously reported that CXCR3 was not expressed on
Tregs in normal kidneys, but was expanded following reperfusion, and inversely correlated with BUN and Scr levels and kidney histology score (17). The present study directly explored the role of CXCR3 in mice renal IRI. It was observed that administration of OE-CXCR3 lentivirus vectors significantly decreased the blood levels of BUN and Scr. KIM-1 is a type 1 transmembrane protein strongly induced by ischemic and toxic insults to kidney, which is undetectable in healthy kidneys, but rises rapidly following injury (23). Nephrin is a specific glomerular podocyte marker, which is reported to be decreased time-dependently starting from $24 \mathrm{~h}$ after renal IRI (24). The results of the present study demonstrated that overexpression of CXCR3 decreased KIM-1 expression and increased Nephrin expression induced by IR, suggesting that CXCR3 exerted a protective role in renal IR, which was further confirmed by the HE and Masson staining. The protective role of CXCR3 may be mediated by a different mechanism than increasing the level of Th1 cells as previously described in $\mathrm{CXCR}^{-1-}$ mice (21). Moreover, silencing and overexpression of CXCR 3 exerted different roles in renal IRI through various mechanisms $(21,22)$. 
To elucidate the mechanism by which CXCR3 protected against renal IRI, the role of CXCR3 in inflammation and oxidative stress was assessed. Overexpression of CXCR3 apparently decreased the blood and kidney levels of pro-inflammatory factors, including IL-6, TNF- $\alpha$ and CCL-2, while increasing the level of IL-10, an anti-inflammatory factor. In addition, CXCR3 upregulation decreased the kidney levels of MDA, and increased SOD and GSH-Px levels. These results illustrated that CXCR3 exerted both anti-inflammatory and anti-oxidative stress roles in kidney IRI. CXCR3 is a receptor of the CXC chemokine subfamily and has been reported to be closely involved in inflammatory diseases (25). For example, CXCR3 promoted steatohepatitis in mice by enhancing inflammation and autophagy (26). CXCR3-deficient mice fed high-fat diet demonstrated reduced mRNA expression levels of pro-inflammatory mediators, such as monocyte chemoattractant protein-1, and promoted the secretion of anti-inflammatory genes, such as Foxp3, IL-10 and arginase-1 in peri-epididymal adipose tissues (27). Although CXCR3 is activated by CXCL9, CXCL10 and CXCL11, growing evidence has identified that the outcome differs when CXCR3 is activated by different ligands $(28,29)$. CXCL9 and CXCL10 are pro-inflammatory, while CXCL11 is anti-inflammatory $(30,31)$. Zohar et al (32) reported that CXCL11 bonded to CXCR3, leading to the increase of FOXP3 $-\mathrm{IL}^{-10^{+}} \mathrm{T}$ regulatory 1 cells and $\mathrm{IL}_{-} 4^{+} \mathrm{Th} 2$ cells, and inflammation repression through activating STAT3 and STAT6. However, the present study did not explore the upstream activator of CXCR3 in kidney IRI. It is hypothesized that CXCL11 binds to CXCR3, resulting in the anti-inflammatory role of CXCR3 in renal IRI; this will be explored in future studies.

Increasing evidence has demonstrated that Tregs are beneficial in the treatment of kidney IRI (11-13). CXCR3 has been considered to be mainly expressed on the surface of pro-inflammatory cells in the past, but our previous study found that CXCR3 was also expressed on Tregs (17). CXCR3 can drive Tregs to migrate to damaged sites $(15,16)$. Knockout of CXCR3 can significantly affect the recruitment of Th1 and Tregs in the lesion site $(33,34)$. Hasegawa et al $(15)$ upregulated CXCR3 expression in Treg cells and then transferred them into recipient mice and assessed their role in the mitigation of experimental GVHD models. The results demonstrated that recipient mice that had undergone transfer of CXCR3-Treg cells demonstrated significant amelioration of GVHD changes in the liver, lung and intestine. Oo et al (16) isolated $\mathrm{CD}^{+} \mathrm{CD} 25^{+} \mathrm{CD} 127^{\text {low }} \mathrm{FoxP} 3^{+}$Tregs (a main type of $\mathrm{T}$ cells in areas of inflammation in human liver disease) from chronically inflamed human liver tissue removed at transplantation and found that these Tregs expressed higher levels of the chemokine receptors CXCR3 and CCR4 compared with blood-derived Tregs and liver-derived Tregs, suggesting that CXCR3 and CCR4 recruited Tregs to sites of inflammation in patients with chronic hepatitis. In the current study, the results demonstrated that CXCR3 overexpression increased the number of Tregs in the injured kidney tissues. Further experiments demonstrated that Tregs deletion abrogated the role of CXCR3 in mitigating kidney IRI, demonstrating that CXCR3 ameliorated kidney IRI through expansion of Tregs. However, Bakheet et al (35) found that administration of AMG487, an antagonist of CXCR3, to mice with collagen-induced arthritis significantly decreased histological inflammatory damage, the percentage of Th1, Th17 and Th22 cells, and increased Tregs. The different tissue contents and conditions may cause this disparity.

Evidence has identified that CXCR3 exerts both pro- and anti-inflammatory roles depending on different ligands. CXCL9 and CXCL10 are pro-inflammatory (30,31), while CXCL11 has anti-inflammatory actions (32). It was hypothesized that CXCL11 binds to CXCR3, resulting in the anti-inflammatory role of CXCR3 in renal IRI. It is intended to verify this in future studies with in vivo and in vitro experiments; for example, by using a co-immunoprecipitation assay to determine whether CXCL11 binds to CXCR3 in vitro and using animal models to study the role of CXCL11 in renal IRI.

In the present study, a non-targeting lentivirus control was applied as the negative control of the OE-CXCR3 lentivirus and it was not examined whether an empty vector had no effect on kidney IRI. The current study excluded the effect of the non-targeting lentivirus control on kidney IRI, and thus, this do not affect the authenticity of the results. However, this is a minor limitation of the present study.

In conclusion, the present study demonstrated that injection of OE-CXCR3 lentivirus into animal models ameliorated kidney IRI by increasing of the number of Tregs, which is a promising method for the prevention and treatment of renal IRI.

\section{Acknowledgements}

Not applicable.

\section{Funding}

The present study was supported by funds from The Chongqing Medical Science Research Project (grant no. 2013-2-021), The General Basic \& Advanced Project of Chongqing Scientific Committee (grant no. cstc2017jcyjAX0419), National Key Clinical Specialist Construction Project of the Ministry of Public Health (grant no. 2011-170) and the Medical Key Subjects in Chongqing Province (grant no. 2007-2).

\section{Availability of data and materials}

All data generated or analyzed during this study are included in this published article.

\section{Authors' contributions}

JC conceived the study and revised the manuscript; WX and JW performed the experiments and were responsible for the draft manuscript; QL and XD performed the data analysis; NW, DC and WG analyzed data and organized the figures. WX, JW and JC confirm the authenticity of all the raw data. All authors read and approved the final manuscript.

\section{Ethics approval and consent to participate}

Animal experiments were carried out at The First Affiliated Hospital of Chongqing Medical University and were approved by the Animal Ethics and Use Committee of the 
First Affiliated Hospital of Chongqing Medical University (approval no. 2017-028).

\section{Patient consent for publication}

Not applicable.

\section{Competing interests}

The authors declare that they have no competing interests.

\section{References}

1. Smith SF, Hosgood SA and Nicholson ML: Ischemia-reperfusion injury in renal transplantation: 3 key signaling pathways in tubular epithelial cells. Kidney Int 95: 50-56, 2019.

2. Hosszu A, Fekete A and Szabo AJ: Sex differences in renal ischemia-reperfusion injury. Am J Physiol Renal Physiol 319 : F149-F154, 2020.

3. Kim M, Park SW, Kim M, Chen SW, Gerthoffer WT, D'Agati VD and Lee HT: Selective renal overexpression of human heat shock protein 27 reduces renal ischemia-reperfusion injury in mice. Am J Physiol Renal Physiol 299: F347-F358, 2010.

4. Jang HR and Rabb H: Immune cells in experimental acute kidney injury. Nat Rev Nephrol 11: 88-101, 2015.

5. Fontenot JD and Rudensky AY: A well adapted regulatory contrivance: Regulatory T cell development and the forkhead family transcription factor Foxp3. Nat Immunol 6: 331-337, 2005

6. Luan H, Wang C, Sun J, Zhao L, Li L, Zhou B, Shao S, Shen X and $\mathrm{Xu} \mathrm{Y:} \mathrm{Resolvin} \mathrm{D1} \mathrm{protects} \mathrm{against} \mathrm{ischemia/reperfu-}$ sion-induced acute kidney injury by increasing treg percentages via the ALX/FPR2 pathway. Front Physiol 11: 285, 2020.

7. Haas J, Schwarz A, Korporal-Kuhnke M, Jarius S and Wildemann B: Myeloid dendritic cells exhibit defects in activation and function in patients with multiple sclerosis. J Neuroimmunol 301: 53-60, 2016.

8. Yazdani M, Khosropanah S, Hosseini A and Doroudchi M: Resting and activated natural tregs decrease in the peripheral blood of patients with atherosclerosis. Iran J Immunol 13: 249-262, 2016

9. Ahmad SF, Zoheir KMA, Ansari MA, Nadeem A, Bakheet SA, Al-Ayadhi LY, Alzahrani MZ, Al-Shabanah OA, Al-Harbi MM and Attia SM: Dysregulation of Th1, Th2, Th17, and T regulatory cell-related transcription factor signaling in children with autism. Mol Neurobiol 54: 4390-4400, 2017.

10. Alroqi FJ and Chatila TA: T regulatory cell biology in health and disease. Curr Allergy Asthma Rep 16: 27, 2016.

11. Bai M, Zhang L, Fu B, Bai J, Zhang Y, Cai G, Bai X, Feng Z, Sun $S$ and Chen X: IL-17A improves the efficacy of mesenchymal stem cells in ischemic-reperfusion renal injury by increasing Treg percentages by the COX-2/PGE2 pathway. Kidney Int 93: $814-825,2018$

12. Gandolfo MT, Jang HR, Bagnasco SM, Ko GJ, Agreda P, Satpute SR, Crow MT, King LS and Rabb H: Foxp3 ${ }^{+}$regulatory $\mathrm{T}$ cells participate in repair of ischemic acute kidney injury. Kidney Int 76: 717-729, 2009.

13. Kinsey GR, Huang L, Vergis AL, Li L and Okusa MD Regulatory T cells contribute to the protective effect of ischemic preconditioning in the kidney. Kidney Int 77: 771-780, 2010.

14. Qin S, Rottman JB, Myers P, Kassam N, Weinblatt M, Loetscher M, Koch AE, Moser B and Mackay CR: The chemokine receptors CXCR3 and CCR5 mark subsets of T cells associated with certain inflammatory reactions. J Clin Invest 101: 746-754, 1998.

15. Hasegawa H, Inoue A, Kohno M, Lei J, Miyazaki T, Yoshie O, Nose M and Yasukawa M: Therapeutic effect of CXCR3-expressing regulatory $\mathrm{T}$ cells on liver, lung and intestinal damages in a murine acute GVHD model. Gene Ther 15 $171-182,2008$

16. Oo YH, Weston CJ, Lalor PF, Curbishley SM, Withers DR, Reynolds GM, Shetty S, Harki J, Shaw JC, Eksteen B, et al: Distinct roles for CCR4 and CXCR3 in the recruitment and positioning of regulatory T cells in the inflamed human liver. J Immunol 184: 2886-2898, 2010.
17. Jun C, Qingshu L, Ke W, Ping L, Jun D, Jie L and Su M: Protective effect of CXCR3(+)CD4(+)CD25(+)Foxp3(+) regulatory T cells in renal ischemia-reperfusion injury. Mediators Inflamm 2015: 360973, 2015

18. Gu J, Sun P, Zhao H, Watts HR, Sanders RD, Terrando N, Xia P, Maze M and Ma D: Dexmedetomidine provides renoprotection against ischemia-reperfusion injury in mice. Crit Care 15: R153, 2011.

19. Zhang Q, Luan H, Wang L, He F, Zhou H, Xu X, Li X, Xu Q, Niki T, Hirashima M, et al: Galectin-9 ameliorates anti-GBM glomerulonephritis by inhibiting Th1 and Th17 immune responses in mice. Am J Physiol Renal Physiol 306: F822-F832, 2014.

20. Livak KJ and Schmittgen TD: Analysis of relative gene expression data using real-time quantitative PCR and the 2(-Delta Delta C(T)) method. Methods 25: 402-408, 2001.

21. Fiorina P, Ansari MJ, Jurewicz M, Barry M, Ricchiuti V, Smith RN, Shea S, Means TK, Auchincloss H Jr, Luster AD, et al: Role of CXC chemokine receptor 3 pathway in renal ischemic injury. J Am Soc Nephrol 17: 716-723, 2006.

22. Zhang C, Zheng L, Li L, Wang L, Li L, Huang S, Gu C, Zhang L, Yang C, Zhu T and Rong R: Rapamycin protects kidney against ischemia reperfusion injury through recruitment of NKT cells. J Transl Med 12: 224, 2014

23. Ichimura T, Bonventre JV, Bailly V, Wei H, Hession CA, Cate RL and Sanicola M: Kidney injury molecule-1 (KIM-1), a putative epithelial cell adhesion molecule containing a novel immunoglobulin domain, is up-regulated in renal cells after injury. J Biol Chem 273: 4135-4142, 1998.

24. Villa L, Buono R, Ferrandi M, Molinari I, Benigni F, Bettiga A, Colciago G, Ikehata M, Messaggio E, Rastaldi MP, et al: Ouabain contributes to kidney damage in a rat model of renal ischemia-reperfusion injury. Int J Mol Sci 17: 1728, 2016.

25. Lacotte S, Brun S, Muller S and Dumortier H: CXCR3, inflammation, and autoimmune diseases. Ann N Y Acad Sci 1173: 310-317, 2009.

26. Zhang X, Han J, Man K, Li X, Du J, Chu ESH, Go MYY, Sung JJY and $\mathrm{Yu}$ J: CXC chemokine receptor 3 promotes steatohepatitis in mice through mediating inflammatory cytokines, macrophages and autophagy. J Hepatol 64: 160-170, 2016.

27. Rocha VZ, Folco EJ, Ozdemir C, Sheikine Y, Christen T, Sukhova GK, Tang EHC, Bittencourt MS, Santos RD, Luster AD, et al: CXCR3 controls T-cell accumulation in fat inflammation. Arterioscler Thromb Vasc Biol 34: 1374-1381, 2014.

28. Altara R, Mallat Z, Booz GW and Zouein FA: The CXCL10/CXCR3 axis and cardiac inflammation: Implications for immunotherapy to treat infectious and noninfectious diseases of the heart. J Immunol Res 2016: 4396368, 2016.

29. Abron JD, Singh NP, Murphy AE, Mishra MK, Price RL, Nagarkatti M, Nagarkatti PS and Singh UP: Differential role of CXCR3 in inflammation and colorectal cancer. Oncotarget 9: 17928-17936, 2018.

30. Karin N, Wildbaum G and Thelen M: Biased signaling pathways via CXCR3 control the development and function of $\mathrm{CD}^{+} \mathrm{T}$ cell subsets. J Leukoc Biol 99: 857-862, 2016.

31. Karin $\mathrm{N}$ and Wildbaum G: The role of chemokines in adjusting the balance between $\mathrm{CD}^{+}$effector $\mathrm{T}$ cell subsets and FOXp3-negative regulatory T cells. Int Immunopharmacol 28: 829-835, 2015.

32. Zohar Y, Wildbaum G, Novak R, Salzman AL, Thelen M, Alon R, Barsheshet Y, Karp CL and Karin N: CXCL11-dependent induction of FOXP3-negative regulatory $\mathrm{T}$ cells suppresses autoimmune encephalomyelitis. J Clin Invest 124: 2009-2022, 2014.

33. Chung AC and Lan HY: Chemokines in renal injury. J Am Soc Nephrol 22: 802-809, 2011.

34. Groom JR and Luster AD: CXCR3 in T cell function. Exp Cell Res 317: 620-631, 2011

35. Bakheet SA, Ansari MA, Nadeem A, Attia SM, Alhoshani AR, Gul G, Al-Qahtani QH, Albekairi NA, Ibrahim KE and Ahmad SF: CXCR3 antagonist AMG487 suppresses rheumatoid arthritis pathogenesis and progression by shifting the Th17/Treg cell balance. Cell Signal 64: 109395, 2019.

This work is licensed under a Creative Commons Attribution-NonCommercial-NoDerivatives 4.0 International (CC BY-NC-ND 4.0) License. 